\title{
Perinatal-Neonatal Management of COVID-19 Infection - Guidelines of the Federation of Obstetric and Gynaecological Societies of India (FOGSI), National Neonatology Forum of India (NNF), and Indian Academy of Pediatrics (IAP)
}

\author{
Deepak Chawla ${ }^{1}$, Dinesh Chirla ${ }^{2}$, SAMir Dalwai ${ }^{3}$, AshoK K DeOrari ${ }^{4}$, Atul Ganatra ${ }^{5}$, AlPesh Gandhi ${ }^{6}$, \\ Nandkishor S Kabra ${ }^{7}$, Praveen Kumar ${ }^{8}$, Pratima Mittal ${ }^{9}$, Bakul Jayant Parekh ${ }^{10}$, M Jeeva SankaR ${ }^{4}$, Tanu \\ Singhal ${ }^{11}$, Sindhu Sivanandan ${ }^{12}$ AND PARIKShit TANK ${ }^{13}$ FOR THe Federation OF ObSTETRIC AND \\ GYNAECOLOGICAL SociETIES OF INDIA (FOGSI), NATIONAL NEONATOLOGY FORUM OF INDIA (NNF) AND INDIAN \\ ACADEMy OF Pediatrics (IAP) \\ From ${ }^{1}$ Department of Neonatology, GMCH, Chandigarh; ${ }^{2}$ Intensive Care Services, Rainbow Children's hospital group; \\ ${ }^{3}$ Department of Pediatrics, Nanavati and Hinduja Hospitals, Mumbai; ${ }^{4}$ Department of Pediatrics, AIIMS, New Delhi; ${ }^{5}$ Dr RJ \\ Ganatra's Nursing Home; ${ }^{6}$ Department of Obstetrics and Gynecology, Arihant Women's hospital, Ahmedabad; ${ }^{7}$ Neonatal Intensive \\ Care Unit, Surya Hospital, Mumbai; ${ }^{8}$ Department of Pediatrics, PGIMER, Chandigarh; ${ }^{9}$ Department of Obstetrics and Gynecology, \\ VMMC and SJH, New Delhi; ${ }^{10}$ BPCH and tertiary care Center, Mumbai; ${ }^{11}$ Department of Pediatrics and Infectious Diseases, \\ Kokilaben Dhirubhai Ambani Hospital \& Medical Research Institute (KDAHMRI), Mumbai; ${ }^{12}$ Department of Neonatology, \\ JIPMER, Puducherry; ${ }^{13}$ Department of Obstetrics and Gynecology, Ashwini Maternity and Surgical Centre, Mumbai; India . \\ Correspondence to: Dr Praveen Kumar, Professor, Department of Pediatrics, PGIMER, Chandigarh, India. \\ drpkumarpgi@gmail.com \\ Submitted: March 30, 2020; Initial review: March 31, 2020; Accepted: April 1, 2020.
}

Justification: During the current rapidly evolving pandemic of COVID-19 infection, pregnant women with suspected or confirmed COVID-19 and their newborn infants form a special vulnerable group that needs immediate attention. Unlike other elective medical and surgical problems for which care can be deferred during the pandemic, pregnancies and childbirths continue. Perinatal period poses unique challenges and care of the mother-baby dyads requires special resources for prevention of transmission, diagnosis of infection and providing clinical care during labor, resuscitation and postnatal period. Process: The GRADE approach recommended by the World Health Organization was used to develop the guideline. A Guideline Development Group (GDG) comprising of obstetricians, neonatologists and pediatricians was constituted. The GDG drafted a list of questions which are likely to be faced by clinicians involved in obstetric and neonatal care. An e-survey was carried out amongst a wider group of clinicians to invite more questions and prioritize. Literature search was carried out in PubMed and websites of relevant international and national professional organizations. Existing guidelines, systematic reviews, clinical trials, narrative reviews and other descriptive reports were reviewed. For the practice questions, the evidence was extracted into evidence profiles. The context, resources required, values and preferences were considered for developing the recommendations. Objectives: To provide recommendations for prevention of transmission, diagnosis of infection and providing clinical care during labor, resuscitation and postnatal period. Recommendations: A set of twenty recommendations are provided under the following broad headings: 1) pregnant women with travel history, clinical suspicion or confirmed COVID-19 infection; 2) neonatal care; 3) prevention and infection control; 4) diagnosis; 5) general questions.

Keywords: Covid-19, Diagnosis, Labor, Management, Newborn, Pregnancy, SARS-CoV-2, Outcome.

Published Online: April 01, 2020; PII: S097475591600154

$\mathrm{C}$ oronaviruses are RNA viruses with glycoprotein spikes that give them a crown like appearance $[1,2]$. Four species have been in circulation for a long time and cause mild respiratory disease. They have a lot of genetic diversity and have jumped the species barrier leading to severe respiratory disease (the SARS virus in 2002-2003 and the MERS virus in 2012-2013). In December, 2019, a novel coronavirus emerged in Wuhan City of Hubei Province in China; this was later termed as SARS-CoV-2 or COVID-19.
This virus has subsequently spread throughout the world causing more than 3.7 million cases and 2,64,000 deaths (till May 07, 2020) [3]. More than 52,000 cases and 1700 deaths have been reported from India [4].

The disease spreads by droplets generated by infected people during sneezing and coughing. These are large droplets that travel for 1-2 $\mathrm{m}$ and settle on surfaces on which the virus can remain alive for hours to days [5]. Infected persons can also spread the infection even before 
the onset of symptoms. Infection is acquired by either inhalation of infected droplets or touching surfaces/ fomites contaminated with the infected droplets and then touching the eyes, nose or mouth. Incubation period varies from 2-14 days with a median of 5 days. The average number of people infected by one infected individual is between two to three. The clinical symptoms are variable, ranging from asymptomatic state to acute respiratory distress syndrome and multi-organ dysfunction. In adults, common symptoms include fever, cough, breathlessness, fatigue, myalgia, headache and sore throat, while vomiting, diarrhea, sneezing and conjunctivitis are uncommon. Current evidence suggests that $80-85 \%$ of cases are mild, $10-15 \%$ are severe with lower respiratory tract involvement, and $5 \%$ are critical, needing ICU care. The fatality rate is reportedly between $2-3 \%$ but can vary from $0.5-10 \%$ depending on the number tested, the percentage of elderly people in the population and availability of critical care support in the hospitals. The severity and fatality are higher in the elderly, especially above the age of 60 (among those aged more than 80 years, fatality rate was $15 \%$ ), and those with comorbidities like heart disease, hypertension, diabetes etc. There is paucity of data on COVID-19 in pregnancy and neonates. Available data suggests that in general the outcome among pregnant women and neonates is good. A large proportion of infected pregnant women are likely to be asymptomatic or have mild symptoms [6]. However, severe disease needing admission to intensive care unit has been reported among pregnant women. Emerging evidence indicates that among women infected with COVID-19 in the third trimester, the risk of vertical transmission is low. Reported clinical features of COVID-19 infection in neonates include fever, lethargy, cough, vomiting and respiratory distress, thus mimicking the presentation of bacterial sepsis $[2,7,8]$.

\section{METHODS}

These guidelines have been developed jointly by the Federation of Obstetric and Gynaecological Societies of India, National Neonatology Forum of India, and Indian Academy of Pediatrics. The GRADE approach recommended by World Health Organization (WHO) was used to develop the guideline [9]. A Guideline development group (GDG) comprising of obstetricians, neonatologists and pediatricians was constituted. The GDG drafted a list of questions which are likely to be faced by clinicians involved in obstetric and neonatal care. An e-survey was carried out amongst a wider group of clinicians to invite more questions and prioritize. Literature search was carried out in PubMed combining the search term ("coronavirus"[MeSH Terms] OR “coronavirus" [All Fields])) AND 2019/12 [PDAT]: 2030 [PDAT]) OR 2019-nCoV [All Fields] OR 2019nCoV [All
Fields] OR COVID-19 [All Fields] OR SARS-CoV-2 [All Fields]) with other key words relevant to the practice question being answered (search updated on 30 April, 2020). In addition, websites of the relevant international and national professional organizations were searched [10-20]. Guidelines, systematic reviews, trials, narrative reviews and other descriptive reports were reviewed. For PICO (participants, intervention, control and outcome) questions, the evidence was extracted into evidence profiles. The context, resources required, values and preferences were considered for developing the recommendations.

\section{OBECTIVES}

The objective of these guidelines is to provide guidance on the short-listed clinical practice questions (Box I).

\section{Pregnant Women}

Of the 3323 articles on the coronavirus infection, 80 addressed the issue in pregnant women. No clinical trials have compared specific care including isolation strategies in pregnant women. A total of 13 studies (12 case series/reports and 1 retrospective cohort study) reported outcome in 113 women with pregnancy and coronavirus infection [21-26]. Due to absence of comparative group it is not possible to estimate the effect of COVID-19 infection in pregnancy. However, almost all pregnant women had mild infection. One died due to severe disease. No clinical trials have compared specific care including isolation strategies in pregnant women. Majority of women in these studies were delivered by $\mathrm{C}$ section; however, in the only case-control study, all controls were also delivered by C-section. Incidence of Csection is high in China, from where all studies have originated, and it is not possible to infer that Covid-19 infection increases the probability of C-section. Literature indicates possibility of higher incidence of fetal distress in infected pregnant women. However, due to small sample size and lack of comparison group, no definite inference can be made. As severe disease and pneumonia have been reported in few reports, pregnant women with infection need to be monitored for respiratory compromise during childbirth.

The treatment of COVID-19 viral infection has been attempted by two approaches. The first approach is the use of a combination of hydroxychloroquine and azithromycin [27]. These drugs are readily available in India and are cost-effective. The other approach has been to use antiviral drugs, some of which are difficult to procure. Hydroxychloroquine in a dose of $600 \mathrm{mg}(200 \mathrm{mg}$ thrice a day with meals) and azithromycin (500 mg once a day) for 10 days has been shown to give virologic cure on 


\section{Box I Short-listed clinical Practice Questions Addressed in the Guidelines}

Pregnant women with travel history, clinical suspicion or confirmed infection

1. What should be the care of pregnant women with history of travel or exposure to a confirmed/suspected case of COVID-19?

2. Which pregnant women need testing for COVID-19?

3. Where in a healthcare facility should a pregnant woman with suspected or active COVID-19 infection deliver?

4. What infection control measures should be undertaken in triage, labor and delivery of pregnant women with active or suspected COVID-19 infection?

5. What should be the method of labor induction and mode of delivery in pregnant women with active or suspected COVID-19 infection?

6. What should be the specific care of pregnant women with active COVID-19 infection?

Neonatal care

7. What precautions should the neonatal resuscitation team take when attending the delivery of a woman with suspected or confirmed COVID-19 infection?

8. What should be the feeding policy for stable neonates born to COVID-19 mothers?

9. Is it necessary to separate the mother and baby if mother is suspected or confirmed to be COVID-19 positive?

10. Should symptomatic neonates needing intensive or special care be nursed in common room NICU/SNCU or isolation facility?

11. What are the special precautions to be taken while providing respiratory support to neonates exposed to COVID-19 infection?

12. In symptomatic neonates, what is the role of specific treatment in case of perinatal exposure and in case of confirmed infection with COVID-19?

Prevention and infection control

13. What should be the specific disinfection practices in NICU /SNCU?

14. When should Personal protective equipment (donning and doffing) be used?

15. What should be the biomedical waste disposal protocol while managing a suspected or confirmed case of COVID-19?

\section{Diagnosis}

16. What should be the testing protocol for neonates born to mothers with suspected or confirmed COVID-19? General questions

17. What should be the visitation policy and preventive measures for visitors during the COVID-19 outbreak?

18. What should be the discharge policy of neonates born to suspected or confirmed COVID-19 mothers?

19. What should be the occupational health policy specific to COVID-19 pandemic?

20. What should be the immunization policy for neonates born to suspected or COVID-19 positive women?

day 6 of treatment in $100 \%$ of treated patients in one study. The study included 20 treated patients with upper and lower respiratory symptoms. In this study, pregnancy was an exclusion criterion. However, as such, both these drugs have been used in pregnancy and during breastfeeding without significant effects on the mother or fetus. Alternative dosage regimens for hydroxychloroquine are to give $400 \mathrm{mg}$ twice a day on day 1 and then $400 \mathrm{mg}$ once a day for the next four days. Chloroquine can also be used as an alternative. The dose is $500 \mathrm{mg}$ twice a day for 7 days. Some authorities recommend that azithromycin should be added only where there is a clinical suspicion of superadded bacterial infection. Lopinavir-ritonavir was the first antiviral combination used to treat COVID-19 infection. However, there was no difference in time to clinical improvement or mortality at 28 days in a randomized trial of 199 patients with severe COVID-19 given lopinavir-ritonavir (400/100 $\mathrm{mg}$ ) twice daily for 14 days in addition to standard care versus those who received standard care alone [28]. Other agents such as remdesivir are being evaluated in randomized trials [29]. Clinicians should follow the latest updated national guidelines released by Indian Council of Medical Research/Ministry of Health and Family Welfare. 


\section{Maternal-fetal Transmission and Neonatal Cases}

Among 707 neonates reported born to Covid-19 positive women, $111(15.7 \%)$ were admitted to NICU, 20 had pneumonia and 3 died. However, in majority of neonates, the reason of admission to NICU was isolation from the infected mother or other morbidities unrelated to COVID-19 infection. Among the 707 births, vertical transmission is suspected in 17 neonates (pooled rate of $2.4 \%$ ), based on virologic and serological reports [30-37]. However, two individual case series have reported higher transmission rates of $7.1 \%$ (3 of 42 ) and 9.1\% (3 of 33) [36].

Of the 17 neonates with suspected vertical transmission, infection was confirmed by a positive RTPCR in 11 (Web Table I). In 3 neonates, infection was suspected based on elevated anti-COVID-19 IgM and IgG levels at birth $[33,34]$. In another 3 neonates, only IgG levels were elevated [33]. In these 6 neonates with elevated antibodies, RT-PCR was repeatedly negative indicating possibility of intrauterine infection of the fetus. Pneumonia was the most common manifestation of infection with 9 neonates of 11 with positive RT-PCR showing clinical and/or radiological evidence of pneumonia. Other clinical features included fever, lethargy and gastrointestinal symptoms. However, the disease was mild in most neonates with only one neonate needing short duration respiratory support and all being discharged alive from the hospital.

Isolation from mother was practiced in all but two of these 17 neonates [35]. Maternal infection was confirmed only in the postnatal period in mothers of these two neonates. Breastfeeding was given by these mothers without wearing masks. In one neonate RT-PCR was positive on day 1 and in second neonate it was positive on day 3 .

Neonatal exposure definitions: As per the Chinese consensus guidelines, neonates are said to be exposed to COVID-19 if they are born to mothers with a history of COVID-19 infection diagnosed within 14 days before delivery or 28 days after delivery, or if the neonate is directly exposed to close contacts with COVID-19 infection (including family members, caregivers, medical staff, and visitors) [38]. They should be managed as patients under investigation (PUI) irrespective of whether they are symptomatic or not.

\section{RECOMMENDATIONS}

Pregnant Women With Travel History, Clinical Suspicion or Confirmed Infection

\section{Recommendation 1}

- Pregnant women with a history of travel or exposure to a confirmed/suspected case of COVID-19 should be isolated by using the ICMR guidelines for nonpregnant adults.

- In the absence of community spread, isolation at the designated facility and in the presence of community spread, isolation by home quarantine may be preferred. For home quarantine, the guidelines issued by ICMR/ MoHFW should be adhered to.

\section{Recommendation 2}

- Testing for pregnant women should be done as per ICMR testing strategy [39].

- In addition, ICMR recommends pregnant women residing in clusters/containment area or in large migration gatherings/evacuation centres from hotspot districts presenting in labor or likely to deliver in next five days should be tested even if asymptomatic [40].

Asymptomatic pregnant women should be tested in the health facilities where they were expected to deliver, and all arrangements should be made to collect and transfer samples to testing facilities. Women should not be referred for lack of testing facility.

\section{Recommendation 3}

- COVID care facilities should be identified in the public and private sector. These would be large multispecialty hospitals with adequate space, infrastructure and logistics. Referral pathways from non-COVID facilities should be well established.

- In such COVID care facilities, three demarcated zones, each housing all the needed equipment and services (wards, labor rooms, operation theatres, neonatal resuscitation areas and mother and neonatal ICU) are required for management of healthy, suspected and confirmed COVID-19 mothers.

The standards and facilities required for infection control in these areas should be same as that for other adults with suspected or confirmed COVID-19.

- Every pregnant woman should be triaged at entry and then allotted into one of the zones depending on the presentation.

- If a woman who delivers in a non-COVID facility turns out to be Covid-19 positive, actions should be taken as per MOHFW's 'Guidelines to be followed on detection of suspect/confirmed COVID-19 case in a non-COVID health facility'[41].

\section{Recommendation 4}

- When providing healthcare to women in labor with confirmed or suspected COVID-19 infection, follow 
standard universal precautions to prevent contact with body fluids. In addition, use personal protective equipment (PPE) to prevent acquiring infection through respiratory droplets. The PPE should include masks such as the N95 and face protection by a face shield or at least goggles.

- Reception and triage should be in the same room that is to be used for admission in labor and delivery. Ideally, this should be a room with negative pressure (If not available, exhaust fans can be installed).

- Keep the room free from any unnecessary items (decorations, extra chairs, etc.) which could act as infected fomites later.

- There should be a restriction on the number of attendants and non-essential staff into the room.

- There should be facilities for health care providers to remove and safely discard PPE at the exit of the room in which the patient is being cared for.

\section{Recommendation 5}

- Mode of delivery in pregnant women infected with COVID-19 should be guided by their obstetric assessment and physiological stability (cardiorespiratory status and oxygenation). COVID-19 infection itself is not an indication for induction of labor or operative delivery.

- Continuous electronic fetal monitoring should be done during labor. If facilities for continuous electronic fetal monitoring are not available, manual monitoring by frequent auscultation of fetal heart rate should be done during the labor, as indicated for a high-risk delivery.

- Adequate equipment and trained healthcare providers should be available for intrapartum monitoring and obstetric interventions as indicated in the separate childbirth facilities for infected pregnant women.

- Oxygenation status of women during labor should be monitored by a pulse oximeter and oxygen therapy should be titrated to maintain oxygen saturation of more than $94 \%$.

\section{Recommendation 6}

- Pregnant women with active COVID-19 infection should be managed with supportive care recommended for non-pregnant adults. Current guidelines by the Government of India do not recommend use of hydroxychloroquine, chloroquine or antiviral drugs in pregnant women.

- Currently recommended national management includes: - oxygen therapy/respiratory support for treatment of hypoxemic respiratory failure, fluid therapy, antibiotics and management of shock.

The choice of specific antiviral therapy is likely to change with rapidly emerging evidence and updated national guidance should be consulted. Updated guidance can be accessed at the website of Ministry of Health and Family Welfare: https://www.mohfw.gov.in/

\section{Neonatal Care}

\section{Recommendation 7}

Recommendations for neonatal resuscitation:

- If possible, resuscitation of neonate can be done in a physically separate adjacent room earmarked for this purpose. If not feasible, the resuscitation warmer should be physically separated from the mother's delivery area by a distance of at least 2 meters. A curtain can be used between the two areas to minimize opportunities for close contact.

- Minimum number of personnel should attend (one person in low risk cases and two in high risk cases where extensive resuscitation may be anticipated) and wear a full set of personal protective equipment including N95 mask.

- Mother should perform hand hygiene and wear triple layer mask.

- The umbilical cord should be clamped promptly and skin to skin contact avoided.

- Delivery team member should bring over the neonate to the resuscitation area for assessment by the neonatal team.

- Neonatal resuscitation should follow standard guidelines. If positive-pressure ventilation is needed, self-inflating bag and mask or a T-piece resuscitator with disposable tubing may be used. Disposable parts should be discarded (even if not used) and reusable equipment/parts should be disinfected after each delivery.

- Routine suction is not indicated for clear or meconium stained amniotic fluid.

- Endotracheal administration of medications should be avoided.

- Indications for intubation shall not change because of maternal COVID-19 status. Plexiglass boxes with access portholes can be used to minimize aerosol spread during intubation and suction.

Disposables like endotracheal tubes, suction catheter, 
orogastric tube, tapes for fixing ET tube, umbilical catheter, syringes placed near the resuscitation area should be discarded even if unused. Reusable equipment should be thoroughly disinfected as per hospital protocol.

- Bathing is not recommended in view of risk of hypothermia and hospital acquired infections.

\section{Recommendation 8}

A. Stable neonates exposed to COVID-19 infection from mothers or other relatives should be roomed-in with their mothers and be exclusively breastfed. For supporting lactation, nurses trained in essential newborn care and lactation management should be provided. A healthy willing family member who is not positive for COVID-19, and has not been in direct contact with suspected or confirmed COVID-19 person and is asymptomatic may be allowed in the room to provide support for breastfeeding and helping in taking care of the neonate.

B. If rooming-in is not possible because of the sickness in the neonate or the mother, the neonate should be fed expressed breast milk of the mother by a nurse or a trained family member who has not been in contact with the mother or other suspected/proven case, provided the neonate can tolerate enteral feeding.

Weak recommendation, based on consensus among experts in the absence of evidence for any beneficial effect or harm in the risk of COVID-19 following direct breastfeeding or expressed breastmilk feeding.

\section{Conditions to be met for safe breastfeeding:}

- Mothers should perform hand hygiene frequently, including before and after breastfeeding and touching the baby.

- Mothers should practice respiratory hygiene and wear a mask while breastfeeding and providing other care to the baby; they should routinely clean and disinfect the surfaces.

- Mothers can express milk after washing hands and breasts and while wearing a mask. If possible, a dedicated breast pump should be provided. If not, it should be decontaminated as per protocol. This expressed milk can be fed to the baby without pasteurization. The collection and transport of EBM to the baby should be done very carefully to avoid contamination.

C. Mothers are not eligible to donate milk in any of the following COVID-19 related situations in addition to standard criteria [42].
- COVID-19 positive donor till she is declared free of infection.

- History of having stayed or transited in a containment zone during the previous 14 days.

- History of close contact with a confirmed or probable case of COVID-19 in previous 14 days.

- Suffering from symptoms like cough, fever, sore throat, running nose till found to be COVID-19 negative on nasopharyngeal sample RT-PCR.

- Person who worked in or attended a health care facility in which a case of COVID-19 infection has been confirmed.

\section{Recommendation 9}

- Healthy neonate may be roomed-in with mother. The mother-baby dyad must be isolated from other suspected and infected cases and healthy uninfected mothers and neonates.

- Direct breastfeeding can be given. Mother should wash hands frequently including before breastfeeding and wear mask. If needed due to neonatal or maternal condition, expressed breast milk may also be fed.

- If safe, early discharge to home followed by telephonic follow-up or home visit by a designated healthcare worker may be considered.

\section{Recommendation 10}

- Neonates who are symptomatic/ sick and are born to a mother with suspected or proven COVID-19 infection should be managed in separate isolation facility.

- This area should be separate from the usual NICU/ SNCU with a transitional area in-between. This isolation facility should preferably have single closed rooms.

- In case enough single rooms are not available, closed incubators (preferred) or radiant warmers could be placed in a common isolation ward for neonates. The neonatal beds should be at a distance of at least 1 meter from one another. Suspected COVID-19 cases and confirmed COVID-19 cases should ideally be managed in separate isolations. If it is not feasible to have separate facilities and the neonates with suspected and confirmed infection are in a single isolation facility, they should be segregated by leaving enough space between the two cohorts.

- The isolation ward should have a separate double door entry with changing room and nursing station. It should be away from routine NICU/SNCU/labor room/ postnatal ward in a segregated area which is not 
frequented by other personnel. The access to isolation ward should be through dedicated lift or guarded stairs.

- Negative air borne isolation rooms are preferred for patients requiring aerosolization procedures (respiratory support, suction, nebulization). If not available, negative pressure can also be created by exhaust fans driving air out of the room.

- Isolation rooms should have adequate ventilation. If room is air-conditioned, ensure 12 air changes/ hour and filtering of exhaust air. These areas should not be a part of the central air-conditioning.

- The doctors, nursing and other support staff working in these isolation rooms should be separate from the ones who are working in regular NICU/SNCU. The staff should be provided with adequate supplies of PPE. The staff also needs to be trained for safe use and disposal of PPE.

If the facilities of isolation intensive care are not available in the hospital where symptomatic or sick newborn is born or referred with COVID-19 infections, the newborn should be immediately shifted to the closest state designated COVID hospital where such facilities are available. Complete safety, PPE policies and precautions must be followed during transport.

\section{Recommendation 11}

- Respiratory support for neonates with suspected/ proven COVID-19 infection is guided by principles of lung protective strategy including use of non-invasive ventilation.

- NIPPV and high flow nasal cannulas should preferably be avoided.

- Healthcare providers should practice contact and droplet isolation and wear N95 mask while providing care in the area where neonates with suspected/ proven COVID-19 infection are being provided respiratory support.

- If intubation is needed:

- Consider use of pre-medication for non-emergent intubation.

- Intubation should be performed by the healthcare worker who is most experienced with airway management.

- Consider use of aerosol box during intubation and suction.

- Consider using in-line suction device.
- Attach a HEPA filter in the path of exhaled gas when using a mechanical ventilator or positive pressure ventilation device.

- The area providing respiratory support should be a negative air pressure area.

\section{Recommendation 12}

- Specific anti-COVID-19 treatment - antivirals or chloroquine/hydroxychloroquine - is NOT recommended in symptomatic neonates with confirmed or suspected COVID-19.

Weak recommendation, based on consensus among experts in the absence of evidence for any beneficial effect or harm with the use of any of the options available.

- Use of adjunctive therapy such as systemic corticosteroids, intravenous gamma globulin and convalescent plasma is NOT recommended in symptomatic neonates with confirmed or suspected COVID-19.

Weak recommendation, based on consensus among experts in the absence of evidence for any beneficial effect or harm with the use of any of the options available.

\section{Prevention and Infection Control}

\section{Recommendation 13}

Disinfection of surfaces in the childbirth/neonatal care areas for patients with suspected or confirmed Coronavirus infection are not different from those for usual Labor room/OT/NICU/SNCU areas and include the following [12]:

- Wear personal protective equipment before disinfecting

- If equipment or surface is visibly soiled first clean with soap and water solution or soaked cloth as appropriate before applying the disinfectant

- $0.5 \%$ sodium hypochlorite (equivalent to $5000 \mathrm{ppm}$ ) can be used to disinfect large surfaces like floors and walls at least once per shift and for cleaning after a patient is transferred out of the area.

- $70 \%$ ethyl alcohol can be used to disinfect small areas between uses, such as reusable dedicated equipment.

- Hydrogen peroxide (dilute $100 \mathrm{ml}$ of $\mathrm{H}_{2} \mathrm{O}_{2} 10 \% \mathrm{v} / \mathrm{v}$ solution with $900 \mathrm{ml}$ of distilled water) can be used for surface cleaning of incubators, open care systems, infusion pumps, weighing scales, standby equipmentventilators, monitors, phototherapy units, and 
shelves. Use $\mathrm{H}_{2} \mathrm{O}_{2}$ only when equipment is not being used for the patient. For ensuring the efficacy of disinfection with $\mathrm{H}_{2} \mathrm{O}_{2}$ use the contact period recommended by manufacturer. Usually a contact period of 1 hour is required.

\section{Recommendation 14}

Minimal composition of a set of PPE for the management of suspected or confirmed cases of COVID-19 infection is provided in Box II.

\section{Recommendation 15}

- Follow routine biomedical waste disposal handling, segregation, transport and final disposal guidelines as prescribed by the Government of India [15].

\section{Diagnosis}

\section{Recommendation 16}

Guidelines on testing of neonates for COVID-19 are provided in Box III.

\section{General Questions}

\section{Recommendation 17}

- Parents and families of the COVID-19 exposed, suspected and infected mothers and neonates should receive informed healthcare. They should be aware of and understand the isolation, monitoring, diagnostic and treatment plans of the mothers/babies and be given a periodic update about the health condition.

- Visitors to both routine and COVID-19 specific childbirth/neonatal care areas should be screened for symptoms of COVID-19 infection.

- Persons (including parents) with suspected or confirmed COVID-19 infection should not be allowed entry in the childbirth/neonatal care area where care to parturient women/sick neonates is being provided.

- For neonates roomed in with mother having suspect/ confirmed COVID-19 infection, one healthy family member following contact and droplet precautions should be allowed to stay with her to assist in baby care activities.

- Visitation policy for COVID-19 infected mother to see her neonate admitted in NICU. Mother may be allowed to visit if

- Resolution of fever without the use of antipyretics for at least 72 hours AND

- Improvement (but not full resolution) in respiratory symptoms AND

- Negative results of a molecular assay for detection of SARS-CoV-2 from at least two consecutive nasopharyngeal swab specimens collected $\geq 24$ hours apart

\section{Recommendation 18}

- Stable neonates exposed to COVID-19 and being roomed-in with their mothers may be discharged at time of mothers' discharge.

Weak recommendation, based on consensus among experts based on the incubation period of SARS-CoV-2 infection in adults and older children.

- Stable neonates in whom rooming-in is not possible because of the sickness in the mother and are being cared by a nurse or a trained family member may be discharged from the facility by $24-48$ hours of age.

Weak recommendation, based on consensus among experts in the absence of evidence for any beneficial effect or harm with early discharge following exposure to COVID-19

\section{Remarks}

- Early discharge to home may be followed by a telephonic follow-up or home visit by a designated healthcare worker.

- Mothers and family members should be counselled regarding the danger signs and advised to report back to the facility if the neonate develops any of the danger signs

Box II Desired Protection and Suggested Personal Protection Equipment for the management of Suspected/Confirmed patient of COVID-19

Respiratory protection

- Triple layered surgical mask

- N95 facemasks are needed when performing an aerosol-generating procedure or in an area where neonates are being provided respiratory support by CPAP device/ventilator.

Eye protection

Goggles (will not be usable by those using vision glasses) or face shield

Body protection

Full-sleeved water-resistant gown including head and complete shoe cover. A single piece head to toe water resistant body cover will be ideal for attending resuscitation in delivery room or OT

Hand protection

Well-fitting gloves 


\section{Box III Guidelines for Testing of Neonates for COVID-19}

\section{Which neonates?}

- History of exposure to COVID-19 positive adult (Irrespective of symptoms):

o Mother had COVID-19 infection within 14 days before birth, or

o History of contact with COVID-19 positive persons (including mother, family members in same household or direct healthcare provider) in postnatal period

- Irrespective of history of exposure:

o Presenting with pneumonia or SARI that requires hospitalization, with onset at more than $48-72 \mathrm{~h}$ of age, unless there is another underlying illness that completely explains the respiratory signs and symptoms.

Features which suggest acute respiratory illness in a neonate are respiratory distress, with or without cough, with or without fever.

When?

- If symptomatic, specimens should be collected as soon as possible

- If asymptomatic, take swab at 48 hours. If neonate's test at 48 hours is negative, repeat test should be done between 5-14 days.

What sample?

Not mechanically ventilated: Upper respiratory nasopharyngeal swab (NP). Collection of oropharyngeal swabs $(\mathrm{OP})$ is a lower priority and if collected should be combined in the same tube as the NP swab.

Mechanically ventilated: Tracheal aspirate sample should be collected and tested as a lower respiratory tract specimen in addition to NP swab.

How to collect?

Upper nasopharyngeal swab

- Use only synthetic fiber swabs with plastic shafts. Do not use calcium alginate swabs or swabs with wooden shafts, as they may contain substances that inactivate some viruses and inhibit PCR testing.

- Insert a swab into nostril parallel to the palate. Swab should reach depth equal to distance from nostrils to outer opening of the ear. Leave swab in place for several seconds to absorb secretions. Slowly remove swab while rotating it.

- Place swabs immediately into sterile tubes containing 2-3 mL of viral transport media. Oropharyngeal swab (e.g., throat swab)Swab the posterior pharynx, avoiding the tongue.Nasopharyngeal wash/aspirate or nasal aspirateCollect 2-3 mL into a sterile, leak-proof, screw-cap sputum collection cup or sterile dry container.Other samples Currently not advised; stool, urine and blood specimens, since the isolation is less reliable than from respiratory specimens. Do not take these specimens for testing (based on current advisory recommendations)

What PPE is needed for sample collection?

Clinicians should wear appropriate personal protective equipment during sampling.

Nasopharyngeal swab

- Hand hygiene

- Disposable single use glove

- Disposable plastic apron

- Surgical facemask

- Eye protection (surgical mask with integrated visor or full-face shield or visor or goggles/safety spectacles)

For any sampling from lower respiratory tract in intubated neonates, a full set of PPE is a must.

- Hand hygiene

- disposable single use glove

- Long sleeved disposable gown

- N95 mask or another respirator mask

- Eye protection

Labelling the sample

Contd... 


\section{Box III continuted}

Label each specimen container with the patient's name, hospital ID number, specimen type and the date the sample was collected. Handle the sample with precautions under biosafety level 3 (BSL-3) conditions until is rendered non-infectious by laboratory.

How to store?

Samples should be collected in viral transport media procured from microbiology laboratory and transported immediately in icepacks. One can use disposable thermocol cartons or plastic bags with ice cubes for in-house transport. If sending to another laboratory, store specimens at $2-8^{\circ} \mathrm{C}$ for up to $72 \mathrm{~h}$ after collection. Storage can be done in a refrigerator dedicated for this purpose. If a delay in testing or shipping is expected, store specimens at $-70^{\circ} \mathrm{C}$ or below. This requires deep freezers.

How to send?

If transporting by shipping, the samples need to be packed as per instructions Guidance for sample Collection, Packaging and Transportation for Novel Coronavirus.

Where to send?

Authorized laboratories (See MOHFW website for latest list )

What test?

Reverse transcriptase PCR is a rapid test for detecting COVID-19

- If the neonate develops any danger signs or becomes unwell during home isolation, he/she should be taken to a designated hospital facility for assessment as well as COVID-19 testing (if indicated)

- Mothers and family members should perform hand hygiene frequently including before and after touching and feeding the baby

- Mothers should practice respiratory hygiene and wear a mask while breastfeeding and providing other care to the baby; they should routinely clean and disinfect all the surfaces.

- If the discharged neonate is positive for COVID-19, uninfected individuals $>60$ years of age (e.g. grandparents) and those with comorbid conditions should not be assigned to provide care if possible.

\section{Recommendation 19}

- Healthcare professionals working in any childbirth or neonatal area should report to their supervisor if they have respiratory or other symptoms suggestive of COVID-19 infection. Such healthcare professional should not be put on clinical duty and should be replaced by a healthy healthcare professional to maintain appropriate patient-provider ratio.

- Healthcare professionals directly involved in the care of patients with suspect/proven COVID-19 infection may consider taking hydroxychloroquine (HCQ) prophylaxis as advised by Government of India, on medical prescription [43]. However, this advisory is based on low-quality evidence and may change in near future.

\section{Recommendation 20}

- Follow routine immunization policy in healthy neonates born to mothers with suspected/proven COVID-19 infection [44].

- In neonates with suspected/proven infection, vaccination should be completed before discharge from the hospital as per existing policy.

\section{Conclusion}

This clinical practice guideline has been jointly developed by FOGSI-IAP-NNF based on the current scientific literature, advisories issued by ICMR and MoHFW and the ground realities of Indian healthcare system. However, our understanding of SARS-Covid-2 virus is incomplete and new insights are being gained everyday. In due course, the guideline shall need to be revised. Readers should check for latest updates.

Disclaimer: The guidelines in this document are based on limited evidence, as is available now. As new evidence accumulates, some of the recommendations may change. Users should use these guidelines in accordance with the latest government regulations and ICMR advisories.

Funding: None. Competing interests: None stated.

\section{REFERENCES}

1. Singhal T. A Review of Coronavirus Disease-2019 (COVID-19). Indian J Pediatr. 2020;87:281-6.

2. Lu Q, Shi Y. Coronavirus disease (COVID-19) and neonate: 
What neonatologist need to know. Journal of Medical Virology. 20 92:564-67.

3. CSSE. COVID-19 Dashboard by the Center for Systems Science and Engineering (CSSE) at Johns Hopkins University (JHU). Available at https://gisanddata. maps.arcgis.com/apps/opsdashboard/index.html\#/ bda7594740fd40299423467b48e9ecf6; 2020.

4. Ministry of Health and Family Welfare. COVID-19 status. Available at https://www.mohfw.gov.in/; 2020.

5. Tang B, Bragazzi NL, Li Q, Tang S, Xiao Y, Wu J. An updated estimation of the risk of transmission of the novel coronavirus (2019-nCov). Infect Dis Model. 2020;5:24855.

6. Sutton D, Fuchs K, D'Alton M, Goffman D. Universal Screening for SARS-CoV-2 in Women Admitted for Delivery. N Engl J Med. 2020/NEJMc2009316. Available at $h t$ tps://www.nejm.org/doi/full/10.1056/NEJMc2009316

7. Kamali Aghdam M, Jafari N, Eftekhari K. Novel coronavirus in a 15-day-old neonate with clinical signs of sepsis, a case report. Infect Dis (Lond). 2020;52:427-9.

8. Wang J, Qi H, Bao L, Li F, Shi Y, National Clinical Research Center for Child Health and Disorders and Pediatric Committee of Medical Association of Chinese People's Liberation Army. A contingency plan for the management of the 2019 novel coronavirus outbreak in neonatal intensive care units. Lancet Child Adolesc Health. 2020;4:258-9.

9. Alonso-Coello P, Schünemann HJ, Moberg J, BrignardelloPetersen R, Akl EA, Davoli M, et al. GRADE Evidence to Decision (EtD) frameworks: A systematic and transparent approach to making well informed healthcare choices. 1: Introduction. BMJ. 2016;353:i2016. Available at https:// www.bmj.com/content/353/bmj.i2016

10. American College of Obstetricians and Gynecologists. Practice Advisory: Novel Coronavirus 2019 (COVID-19). Available from: https://www.acog.org/Clinical-GuidanceandPublications/Practice-Advisories/Practice-AdvisoryNovel-Coronavirus 2019? Accessed on March 25, 2020.

11. Centers for Disease Control. Interim Clinical Guidance for Management of Patients with Conûrmed Coronavirus Disease (COVID-19). Available from: https:// www.cdc.gov/coronavirus/2019-ncov/hcp/clinicalguidance-management-patients.html. Accessed March 20, 2020.

12. National Guidelines for Infection Prevention and Control In Healthcare Facilities, Ministry of Health and Family Welfare, Available from: https://www.mohfw.gov.in/. Accessed March 25, 2020.

13. Favre G, Pomar L, Qi X, Nielsen-Saines K, Musso D, Baud D. Guidelines for pregnant women with suspected SARSCoV-2 infection. Lancet Infect Dis. 2020 Mar;S1473309920301572. Available at: https://www.t helancet.com/pdfs/journals/laninf/PIIS1473-3099(20) 30157-2.pdf. Accessed on March 25, 2020.

14. Guidance for sample Collection, Packaging and Transportation for Novel Coronavirus. Available from: https://www.mohfw.gov.in/pdf/5Sample\%20collection packaging\%20\% 202019-nCoV.pdf. Accessed on March 25,2020 .

15. Guidelines for handling, treatment and disposal of waste generated during treatment, diagnosis and quarantine of COVID-19 patients. March 2020 Published by Central Pollution Control Board, Parivesh Bhawan, New Delhi110032. Available from: https://www.imageima.org/files/ New-CPCB-Guidelines-for-COVID-19-Biomedical-WasteManagement.pdf

16. Interim Guidance for Healthcare Facilities: Preparing for Community Transmission of COVID-19 in the United States. Available from: URL:https://www.cdc.gov/ coronavirus/2019-ncov/healthcare-facilities/guidancehcf.html. Accessed March 20, 2020.

17. World Health Organization. Clinical management of severe acute respiratory infection (SARI) when COVID-19 disease is suspected. Interim Guidance. 2020. Available from:https://www.who.int/publications-detail/clinicalmanagement-of-severe-acute-respiratory-infection-whennovel-coronavirus-(ncov)-infection-is-suspected. Accessed on March 25, 2020.

18. World Health Organization. Water, sanitation, hygiene, and waste management for the COVID-19 virus: Technical brief. 2020. Available from: https://www.who.int/ publications-detail/water-sanitation-hygiene-and-wastemanagement-for-covid-19. Accessed on March 25, 2020.

19. Royal College of Obstetricians and Gynaecologists (RCOG). Coronavirus infection and pregnancy : Information for pregnant women and their families. Available from: https://www.rcog.org.uk/globalassets/ documents/guidelines/coronavirus-covid-19-infectioninpregnancy-v3-20-03-18.pdf. Accessed on March 25, 2020.

20. RCPCH Guidelines: COVID-19 - guidance for paediatric services. Available from: https://www.rcpch.ac.uk/ resources/covid-19-guidance-paediatric-services \#working-in-neonatal-settings. Accessed on March 25, 2020.

21. Chen H, Guo J, Wang C, Fan Luo, Xuechen Yu, Wei Zhang, et al. Clinical characteristics and intrauterine vertical transmission potential of COVID-19 infection in nine pregnant women: a retrospective review of medical records. Lancet. 2020;395:809-15.

22. Li Y, Zhao R, Zheng S, Chen X, Wang J, Sheng X, et al. Lack of vertical transmission of severe acute respiratory syndrome Coronavirus 2, China. Emerg Infect Dis. 2020;26(6). Available from: https://wwwnc.cdc.gov/eid/ article/26/6/20-0287_article

23. Liu D, Li L, Wu X, Dandan Zheng Jiazheng Wang, Lian Yang, et al. Pregnancy and Perinatal Outcomes of Women With Coronavirus Disease (COVID-19) Pneumonia: A Preliminary Analysis [published online ahead of print, 2020 Mar 18]. Am J Roentgenol. 2020;1-6. Available from: https://www.ajronline.org/doi/full/10.2214/AJR.20.23072. Accessed on March 25, 2020.

24. Liu W, Wang Q, Zhang Q, Chen L, Chen J, Zhang B, et al. Coronavirus Disease 2019 (COVID-19) During Pregnancy: A Case Series. 2020 Feb 25 [cited 2020 Mar 21]; Available from: https://www.preprints.org/manuscript/202002. 0373 /v1. Accessed on March 25, 2020.

25. Liu Y, Chen H, Tang K, Guo Y. Clinical manifestations and outcome of SARS-CoV-2 infection during pregnancy. J 
Infect. 2020 Mar 4; Available from: https://www. journalofinfection.com/article/S0163-4453(20)30109-2/ fulltext. Accessed on March 25, 2020.

26. Qi Lu, Yuan Shi, Coronavirus disease (COVID-19) and neonate: what neonatologist need to know; J Med Virol. 2020. Available from: https://onlinelibrary.wiley.com/doi/ full/10.1002/jmv.25740. Accessed on March 25, 2020.

27. Gautret P, Lagier JC, Parola P, Hoang VT, Meddeb L, Mailhe $\mathrm{M}$, et al. Hydroxychloroquine and Azithromycin as a treatment of COVID-19: results of an open label nonrandomized clinical trial. Int J Antimicrob Agents. 2020 Mar 20:105949. Available from: https://www.ncbi. nlm.nih.gov/pmc/articles/PMC7102549/. Epub ahead of print Accessed on March 25, 2020.

28. Cao B, Wang Y, Wen D, Liu W, Wang J, Fan G, et al. A Trial of Lopinavir-Ritonavir in Adults Hospitalized with Severe Covid-19. N Engl J Med. 2020. Available from: https:// www.nejm.org/doi/pdf/10.1056/NEJMoa 2001282. Accessed on March 25, 2020.

29. Li Z, Wang X, Cao D, Sun R, Li C, Li G. Rapid review for the anti-coronavirus effect of remdesiv. Drug Discov Ther. 2020;14(2):73-76.

30. Carosso A, Cosma S, Borella F, Marozio L, Coscia A, Ghisetti V, et al. Pre-labor anorectal swab for SARS-CoV-2 in COVID-19 pregnant patients: Is it time to think about it? European Journal of Obstetrics \& Gynecology and Reproductive Biology. 2020;S0301211520302025.

31. Zamaniyan M, Ebadi A, Mir SA, Rahmani Z, Haghshenas M, Azizi S. Preterm delivery in pregnant woman with critical COVID-19 pneumonia and vertical transmission. Prenatal Diagnosis. 2020. Available from : https:// www.pubfacts.com/search/Prenatal + Diagnosis\% 0D\%09+\%0D\%5BJournal\%5D. Accessed May 2, 2020

32. Alzamora MC, Paredes T, Caceres D, Webb CM, Valdez LM, Rosa ML. Severe COVID-19 during Pregnancy and Possible Vertical Transmission. Am J Perinatol.; 2020; Apr 18. Available at https://www.thieme-connect.com/products/ ejournals/abstract/10.1055/s-0040-1710050.

33. Zeng $\mathrm{H}, \mathrm{Xu} \mathrm{C}$, Fan J, Tang Y, Deng Q, Zhang W, et al. Antibodies in Infants Born to Mothers With COVID-19 Pneumonia. JAMA. 2020 Available at: https:// jamanetwork.com/journals/jama/fullarticle/2763854.

34. Dong L, Tian J, He S, Zhu C, Wang J, Liu C, et al. Possible Vertical Transmission of SARS-CoV-2 From an Infected Mother to Her Newborn. JAMA. 2020; Available at: $h$ ttps:/ /jamanetwork.com/journals/jama/fullarticle/2763853.

35. Zeng L, Xia S, Yuan W, Yan K, Xiao F, Shao J, et al. Neonatal Early-Onset Infection With SARS-CoV-2 in 33 Neonates Born to Mothers With COVID-19 in Wuhan, China. JAMA Pediatr. 2020; Available at : https:// jamanetwork.com/journals/jamapediatrics/fullarticle/ 2763787.

36. Wang S, Guo L, Chen L, Liu W, Cao Y, Zhang J, et al. A Case Report of Neonatal 2019 Coronavirus Disease in China. Clin Infect Dis. 2020; Available at : https:// www.ncbi.nlm.nih.gov/pmc/articles/PMC7108144/

37. Yu N, Li W, Kang Q, Xiong Z, Wang S, Lin X, et al. Clinical features and obstetric and neonatal outcomes of pregnant patients with COVID-19 in Wuhan, China: A retrospective, single-centre, descriptive study. The Lancet Infectious Diseases 2020;20:559-64.

38. Wang L, Shi Y, Xiao T, Fu J, Feng X, Mu D, et al. Chinese expert consensus on the perinatal and neonatal management for the prevention and control of the 2019 novel coronavirus infection (First edition). Ann Transl Med. 2020;8:47. Available from: http://atm.amegroups.com/ article/view/35751/html. Accessed on March 25, 2020.

39. Indian Council of Medical Research. Department of Health Research. Strategy for COVID19 testing in India (Version 4, dated 09/04/2020). Available at: https://www.icmr. gov.in/pdf/covid/strategy/Strategey_for_COVID19_Test_ v4_09042020.pdf. Accessed on April 15, 2020.

40. Indian Council of Medical Research. Department of Health Research. Strategy for COVID19 testing for pregnant women in India (Version 1, dated 20/04/2020) Available at https://www.icmr.gov.in/pdf/covid/strategy/COVID19_ Testing_Strategy_for_Pregnant_Women.pdf. Accessed on April 22, 2020

41. Ministry of Health and Family Welfare Government of India. Guidelines to be followed on detection of suspect/ confirmed COVID-19 case in a non-COVID Health Facility.2020. Available at https://www.mohfw.gov.in/pdf/ Guidelinestobefollowedondetectionofsuspectorconfirmed COVID19 case.pdf. Accessed on May 1,2020.

42. India HMBA, Infant \& Young Child Feeding Chapter of Indian Academy of Paediatrics, PATH. Guidance for use of Human Milk in India in the context of COVID-19 Pandemic. 2020. Available at : https://www.path.org/ resources/guidance-use-human-milk-india-context-covid19/.

43. Indian Council of Medical Research. Department of Health Research. Recommendation for empiric use of hydroxychloroquine for prophylaxis of SARS-CoV-2 infection. Available at https://www.icmr.gov.in/pdf/covid/techdoc/ HCQ_Recommendation_22March_final_MM_V2.pdf. Accessed on March 27,2020

44. IAP Committee on Vaccines \& Immunization. ACVIP Guidelines on Immunizations during COVID 19 Pandemic. 2020. Available at: https://iapindia.org/pdf/FINAL-IAPCOVID-19-Pandemic-Immunization-Activities.pdf. 
Annexure I

\section{Use of Personal Protective Equipment}

\section{Sequence of Donning}

Before wearing the PPE for managing a suspected or confirmed COVID-19 case, proper hand hygiene should be performed. The gown should be donned first. The mask or respirator should be put on next and properly adjusted to fit; remember to fit check the respirator. The goggles or face shield should be donned next and the gloves are donned last. Keep in mind, the combination of PPE used, and therefore the sequence for donning, will be determined by the precautions that need to be taken.

\section{Steps in Removing PPE (Doffing)}

Wearing the PPE correctly will protect the healthcare worker from contamination. After the patient has been examined or desired procedure is performed, the removal of the PPE is a critical and important step that needs to be carefully carried out in order to avoid self-contamination because the PPE could by now be contaminated.

1. The gloves are removed first because they are considered a heavily contaminated item. Use of alcohol-based hand disinfectant should be considered before removing the gloves. Dispose of the gloves in a biohazard bin.

2. After the removal of gloves, hand hygiene should be performed, and a new pair of gloves should be worn to further continue the doffing procedure. Using a new pair of gloves will prevent self-contamination. Unbuttoning of the backside of the gown, performed by an assistant. Removal of gown to be performed by grabbing the back side of the gown and pulling it away from the body. Single-use gowns can now be disposed of; reusable gowns have to be placed in a bag or container for disinfection

3. After the gown, the goggles should be removed and either disposed if they are single-use, or placed in a bag or container for disinfection. In order to remove the goggles, a finger should be placed under the textile elastic strap in the back of the head and the goggles taken off. Touching the front part of the goggles, which can be contaminated, should be avoided. If goggles with temples are used, they should be removed as per manufacturer's recommendations.

4. The respirator/ mask should be removed next. In order to remove the respirator/mask, a finger or thumb should be placed under the straps in the back and the respirator taken off. The respirator (or the surgical mask) should be disposed of after removal. It is important to avoid touching the respirator/mask with the gloves (except for the straps) during its removal.

5. The last PPE items that should be removed are the new set of gloves that were worn after disposal of the contaminated gloves. Use of alcohol-based solution should be considered before removing the gloves. The gloves should be removed Dispose of the gloves in a biohazard bin.

6. After glove removal, hand hygiene should be performed.

\section{Guideline Development Group (Alphabetical)}

Chairperson: Praveen Kumar, Professor, Department of Pediatrics, PGIMER, Chandigarh.

Deepak Chawla, Department of Neonatology, GMCH Chandigarh; Dinesh Chirla, Intensive Care Services, Rainbow Children's hospital group; Samir Dalwai (National Joint Secretary IAP), Pediatrician, Nanavati and Hinduja Hospitals, Mumbai; Ashok K Deorari (President NNF), Department of Pediatrics, AIIMS, New Delhi; Atul Ganatra (Vice-President FOGSI), Dr. $R J$ Ganatra's Nursing Home, Mumbai; Alpesh Gandhi (President FOGSI), Department of Obstetrics \&

\section{Annexure II}

Gynecology, Arihant Women's hospital, Ahmedabad; Nandkishor S Kabra, Surya Hospital, Mumbai; Pratima Mittal, Deppartment of Obstetrics \& Gynecology, VMMC and SJH, New Delhi; Bakul Jayant Parekh (National President IAP), BPCH and tertiary care Center, Mumbai; M Jeeva Sankar, Deppartment of Pediatrics, AIIMS, New Delhi; Tanu Singhal, Deppartment of Pediatrics and Infectious Diseases, KDAHMRI, Mumbai; Sindhu Sivanandan, Deppartment of Neonatology, JIPMER, Puducherry; Parikshit Tank (Joint Treasurer, FOGSI), Ashwini Maternity and Surgical Centre, Mumbai. 